\title{
Preparation and Evaluation of Medicinal Carbon Oral Films
}

\author{
Yoko SAKudA, ${ }^{a}$ Akihiko Ito, ${ }^{* a, b}$ Masanaho SAsatsu, ${ }^{a}$ and Yoshiharu MAchidA ${ }^{a}$ \\ ${ }^{a}$ Department of Drug Delivery Research, Hoshi University; 2-4-41 Ebara, Shinagawa-ku, Tokyo 142-8501, Japan: and \\ ${ }^{b}$ Department of Medicinal Therapy Research, Meiji Pharmaceutical University; 2-522-1 Noshio, Kiyose, Tokyo \\ 204-8588, Japan. Received August 31, 2009; accepted January 13, 2010; published online January 14, 2010
}

\begin{abstract}
Medicinal carbon (MC) films, which can be taken more easily than other dosage forms, were prepared using sodium carboxymethyl cellulose (CMC), hydroxypropylmethyl cellulose (HPMC) and alginic acid sodium (ALG) as film base materials. Brilliant blue FCF (BB) was used as a model drug. The films containing MC had sufficient strength and disintegration time, but their ability to adsorb BB was clearly inhibited compared to that of MC in powder form. When ALG was used as the film base, the BB adsorption capacity of MC film was approximately $50 \%$ of that of MC powder. In an attempt to improve this adsorption ability, two saccharides, sorbitol (SOR) and maltitol (MT), were separately added to MC at a mixing ratio of $1: 1$ by weight. When ALG was the film base, MC films containing SOR or MT showed rapid adsorption profiles and had greatly increased capacities for BB adsorption compared with films containing MC alone. SOR was superior to MT as an additive, though both gave MC-containing films a BB adsorption capacity almost equal to that of MC powder after $24 \mathrm{~h}$, and physical mixtures tended to have better BB adsorption capacities than pre-treatment mixture. In addition, both SOR and MT tended to increase vertical strength of films, but neither additive in either type of mixture had a clear effect on disintegration time. When CMC or HPMC was used as the film base, on the other hand, the addition of SOR or MT caused hardly any improvement in adsorption ability. The above results demonstrate that ALG is useful as a film base material for the preparation of MC films, and that MC films with sufficient strength and adsorption capacities equal to those of MC powders can be produced using a physical mixture of MC and SOR on an ALG base.
\end{abstract}

Key words medicinal carbon powder; film; saccharide; adsorption ability; water soluble polymer; alginic acid sodium

Medicinal carbon (MC) is used clinically as a potent adsorption agent in treating such conditions as intoxication caused by the ingestion of toxic chemicals or other toxins, drug overdose, and the formation of harmful metabolites in the gastrointestinal tract. ${ }^{1-5)}$ It has also been reported to display strong potential to adsorb an endotoxin related to serious symptoms of food poisoning, ${ }^{6}$ and it has been used for removing the waste products that build up as a result of chronic kidney disease. ${ }^{7)}$

MC is usually administered in a powder, granule or capsule form, but many patients report problems with swallowing it in these forms: powder can adhere to the throat and diffuse throughout the mouth, granules can cause pain by entering the space between a denture and the gums, and capsules can adhere to the throat or the esophagus. In an investigation into the ease of swallowing these of oral dosage forms, patients reported that they disliked all three, but that powder was the least convenient. ${ }^{8}$ These problems often lead to patient non-compliance. To avoid these issues, caregivers often prefer to administer $\mathrm{MC}$ in tablet form; not surprisingly, patients also prefer to take MC in tablet form. ${ }^{9)}$ Accordingly, in two previous studies, we investigated the administration of $\mathrm{MC}$ in a compact dosage form, and reported that MC tablets possessing the full adsorption capacity of MC could be made by means of wet granule compression using saccharides as binding agents. ${ }^{10,11)}$ Although most patients can take tablets more easily than powders, granules or capsules, patients with reduced swallowing ability often have trouble taking tablets, and patients who must limit their water consumption also require an alternative dosage form. In this study, therefore, we investigated the feasibility of MC-containing films as a new dosage form for such patients.

\section{Experimental}

Materials Medicinal carbon (MC) was purchased from Kenei Pharmaceutical Co., Ltd. (Osaka, Japan) as a fine powder and used in the experiments without sieving. Sodium carboxymethyl cellulose (CMC) and brilliant blue FCF (BB) were obtained from Wako Pure Chemical Industries Ltd. (Osaka, Japan). Hydroxypropylmethyl cellulose (HPMC) and alginic acid sodium (ALG) were obtained from Sigma-Aldrich (St. Louis, MO, U.S.A.). Maltitol (MT) and sorbitol (SOR) were obtained from Towa Chemical Co., Ltd. (Osaka, Japan). All other chemicals were reagent-grade.

Preparation of Films CMC, HPMC and ALG were tested as potential film bases. Each of these water-soluble polymers was dissolved to $4 \%(\mathrm{w} / \mathrm{v})$ or $2 \%(\mathrm{w} / \mathrm{v})$ aqueous solution. Fifty milliliters of each solution was then added to $2 \mathrm{~g}$ of $\mathrm{MC}$ powder, either alone or combined in a pre-treatment (PRE) or physical mixture (PM) with $2 \mathrm{~g}$ of either MT or SOR. Each mixture was mixed sufficiently with a magnetic stirrer. The mixture was then cast onto partitioned teflon board using the solution casting method, then dried sufficiently at room temperature. Each of the resulting films was a square about $2.0 \times 2.0 \mathrm{~cm}$ in size which contained about $12.5 \mathrm{mg}$ of MC.

PRE were produced according to the following method. Thirty milliliters of water was added to $2 \mathrm{~g}$ of MC and $2 \mathrm{~g}$ of MT or SOR. The mixture was mixed sufficiently and dried at $60^{\circ} \mathrm{C}$ overnight.

This study used PRE and PM of MC and saccharides, because granules prepared in previous studies by the wet granule method using saccharides as binding agents preserved the adsorption capacity of MC. ${ }^{12,13)}$

Measurement of the Physical Characteristics of Films The strength of the films was measured vertically with a Fudoh rheometer (Rheotech, Tokyo, Japan) as shown in Fig. 1a. Disintegration time was measured using a modified disintegration apparatus as shown in Fig. 1b; briefly, a film was clipped onto the arm of a Model NT-60H disintegration tester (Toyama Sangyo Co., Ltd., Osaka, Japan), which moved the film in and out of a $37{ }^{\circ} \mathrm{C}$ purified water bath (a total distance of $5.5 \mathrm{~cm}$ ) at 30 strokes per minute. The length of time required for the film to disintegrate was recorded as the disintegration time. Each prepared film containing MC-only was about 120 $150 \mu \mathrm{m}$ thick and weighed about $20-30 \mathrm{mg}$, while each prepared film containing a PRE or a PM was about $150-200 \mu \mathrm{m}$ thick and weighed about $40-50 \mathrm{mg}$; these estimates are based on the actual measurements of ten films of each type. The thickness of the films was measured with a dogmatic micrometer (Mitutoyo Co., Ltd., Kawasaki, Japan).

Brilliant Blue Adsorption Experiments Twenty-five milligrams of MC, either in powder form or contained in one of the types of film described above, was added to $50 \mathrm{ml}$ of $0.01 \%(\mathrm{w} / \mathrm{v}) \mathrm{BB}$ solution, and the mixture was 
a) Vertical strength

b) Disintegration time

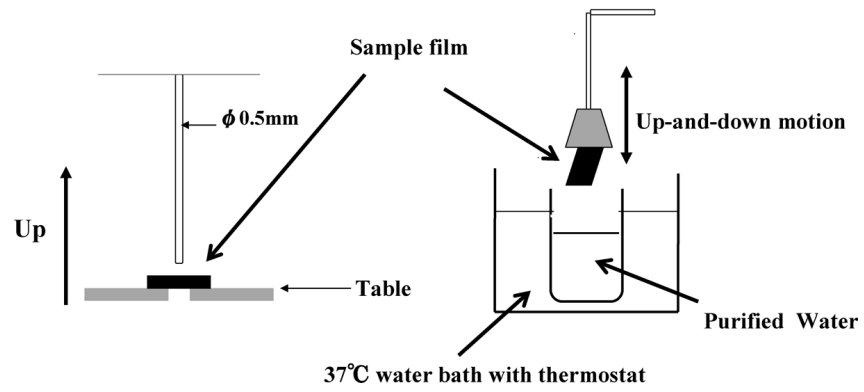

Fig. 1. Measurement Methods of a) Vertical Strength and b) Disintegration Time of MC Films Containing MC Alone

stirred with a magnetic stirrer at $37^{\circ} \mathrm{C}$ in a model BW200 shaking bath (Yamato Scientific Co., Ltd., Tokyo, Japan). At appropriate time points, $1 \mathrm{ml}$ samples were withdrawn and centrifuged at $3000 \mathrm{rpm}$ for $10 \mathrm{~min}$ (Kubota 5200 centrifuge, Kubota Tokyo, Japan). The supernatant was diluted with water and the concentration of free BB was measured using UV spectrophotometry at $629 \mathrm{~nm}$. The difference between the concentrations of free BB and total $\mathrm{BB}$ was recorded as the amount of $\mathrm{BB}$ that had been adsorbed by the $\mathrm{MC}$, i.e., the adsorption capacity of each dosage form.

Statistical Analysis All data are shown as the mean \pm S.D. Statistical differences were analyzed using the Tukey-Kramer test for multiple comparisons. The level of significance was taken as $p<0.05$.

\section{Results and Discussion}

Evaluation of MC Films Prepared Using MC Powder Alone The vertical strength and disintegration time of $\mathrm{MC}$ films composed of $4 \%(\mathrm{w} / \mathrm{v}) \mathrm{CMC}, 4 \%(\mathrm{w} / \mathrm{v}) \mathrm{ALG}$ or $2 \%$ (w/v) HPMC solution mixed with MC powder alone are shown in Table 1. MC/CMC film was the strongest, withstanding a vertical pressure of $84.2 \mathrm{~g}$; in contrast, vertical strengths of MC/HPMC and MC/ALG films were both below $50 \mathrm{~g}$. MC/HPMC film had the longest disintegration time, requiring $116.3 \mathrm{~s}$, while $\mathrm{MC} / \mathrm{CMC}$ and $\mathrm{MC} / \mathrm{ALG}$ film had approximately equivalent disintegration times, in the range of $30-40 \mathrm{~s}$. No certain relationship between these properties and either the thicknesses or the weights of the films was observed.

$\mathrm{BB}$ adsorption experiments were performed on each type of prepared film and on MC powder. The results are shown in Fig. 2. MC powder adsorbed BB rapidly, taking up approximately $66 \%$ of it in approximately $4 \mathrm{~h}$, then reaching a plateau; compared with MC powder, all types of film clearly showed inhibited BB adsorption capacity: MC/ALG film exhibited a slow $\mathrm{BB}$ adsorption profile, taking up only about $30 \%$ of the $\mathrm{BB}$ in $4 \mathrm{~h}$, while $\mathrm{MC} / \mathrm{CMC}$ film and MC/HPMC film adsorbed hardly any BB; taking up less than $10 \%$ in the same interval. We assume that the adsorption capacity of MC films is inhibited because polymer film base fills many of the MC's pores, and that the variation in adsorption capacity among films with different polymer bases is caused by differences in the polymers' solubility and viscosity. It seems likely that differences in the polymers' solubility and viscosity associated with the degree of freeing $\mathrm{MC}$ pores. For example, $\mathrm{MC} / \mathrm{ALG}$ film adsorbs more BB because ALG dissolves more rapidly than the other polymers do, freeing $\mathrm{MC}$ pores in the process, and because ALG has a low viscosity.

Although MC films composed of CMC, ALG or HPMC mixed with $\mathrm{MC}$ powder alone have sufficiently high strengths
Table 1. Physical Properties of MC Films Prepared Using MC Alone

\begin{tabular}{ccc}
\hline \hline Film & Vertical strength $(\mathrm{gf})^{a)}$ & ${\text { Disintegration time }(\mathrm{s})^{b)}}^{b}$ \\
\hline MC/CMC & $84.2 \pm 26.8^{* * * *}$ & $38.5 \pm 14.5$ \\
MC/ALG & $24.4 \pm 5.5$ & $30.7 \pm 5.5$ \\
MC/HPMC & $49.4 \pm 10.2$ & $116.3 \pm 43.7 *, * * *$ \\
\hline
\end{tabular}

a) Mean \pm S.D. $(n=5) . \quad$ b) Mean \pm S.D. $(n=6) . * p<0.05$ compared with MC/ALG, $* * p<0.05$ compared with MC/HPMC, $* * * p<0.05$ compared with MC/CMC.

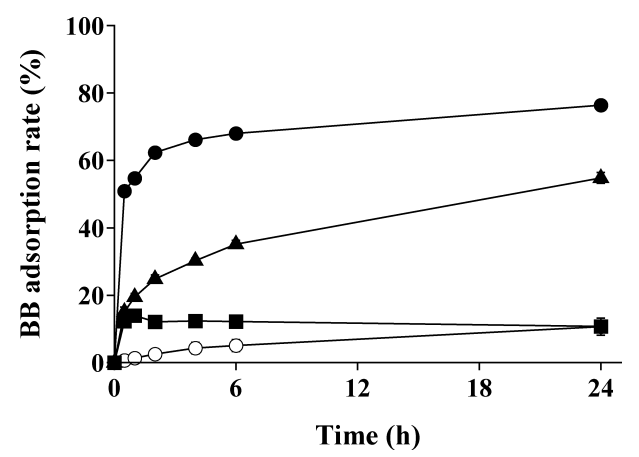

Fig. 2. Brilliant Blue Adsorption Profiles of MC Films Containing MC Alone

- MC powder, $\mathbf{M C} / \mathrm{CMC}$ film, $\boldsymbol{\Delta} \mathrm{MC} / \mathrm{ALG}$ film, $\bigcirc \mathrm{MC} / \mathrm{HPMC}$ film. Each point represents mean \pm S.D. $(n=3)$.

and low disintegration times, their capacity to adsorb $\mathrm{MC}$ is markedly inhibited. Because the main effect of $\mathrm{MC}$ is so greatly diminished, this type of film is not useful.

Evaluation of MC Films Prepared Using Pre-treatment Mixtures and Physical Mixtures of MC Powder and Saccharides In our research on MC tablet preparation methods, we have reported that mixing $\mathrm{MC}$ with water-soluble polymers inhibited MC's adsorption capacity, but that $\mathrm{MC}$ tablets prepared by the wet granule compression method using such saccharides as sorbitol (SOR) and maltitol (MT) as binders retained MC's adsorption capacity. ${ }^{12)}$ Therefore, in an attempt to improve the ability of MC films to adsorb BB, we added SOR and MT to the film mixtures. $4 \%$ (w/v) CMC, $4 \%(\mathrm{w} / \mathrm{v})$ ALG or $2 \%(\mathrm{w} / \mathrm{v})$ HPMC solution was added to a PRE or a PM of MC powder and SOR or MT; films were then prepared according to the method described above.

The results of $\mathrm{BB}$ adsorption experiments on each type of prepared film are shown in Fig. 3. The BB adsorption capacity of $\mathrm{MC} / \mathrm{CMC}$ film was not improved by the addition of SOR or MT in either type of mixture. All MC/CMC films exhibited similar $\mathrm{BB}$ adsorption profiles, and the adsorption capacities of all MC/CMC films with saccharides were below 10\% (Fig. 3a). The BB adsorption capacity of MC/HPMC film was slightly increased by the addition of SOR or MT, but these film's adsorption rates were only approximately $30 \%$ after $24 \mathrm{~h}$ (Fig. 3b). Furthermore, neither the saccharide type nor the mixture type had a significant effect on the adsorption capacities of films with CMC or HPMC bases. In contrast, the $\mathrm{BB}$ adsorption capacity of $\mathrm{MC} / \mathrm{ALG}$ film and the rapidity of its $\mathrm{BB}$ adsorption profile were appreciably increased by the addition of SOR or MT. MC/ALG films containing $\mathrm{PM}$ tended to have better $\mathrm{BB}$ adsorption capacities than MC/ALG films containing PRE (Fig. 3c).

The $\mathrm{BB}$ adsorption rates of $\mathrm{MC}$ powder, $\mathrm{MC} / \mathrm{ALG}$ film containing $\mathrm{MC}$ alone (MC-only film), and $\mathrm{MC} / \mathrm{ALG}$ films 
containing physical mixtures of MC and SOR (MC/SOR-PM film) or MT (MC/MT-PM film) are shown in Table 2. Although all types of MC film adsorbed BB more slowly than MC powder did, MC/SOR-PM and MC/MT-PM films exhibited higher $\mathrm{BB}$ adsorption rates than $\mathrm{MC}$-only film did $(p<0.05)$. MC/SOR-PM film exhibited a higher BB adsorption rate than MC/MT-PM film did $(p<0.05)$, and adsorbed BB nearly as well as MC powder: after $6 \mathrm{~h}$, its adsorption capacity was almost equal to that of $\mathrm{MC}$ powder, and after $24 \mathrm{~h}$, its $\mathrm{BB}$ adsorption capacity was equal to that of $\mathrm{MC}$ powder and approximately 1.4 times that of MC-only film.
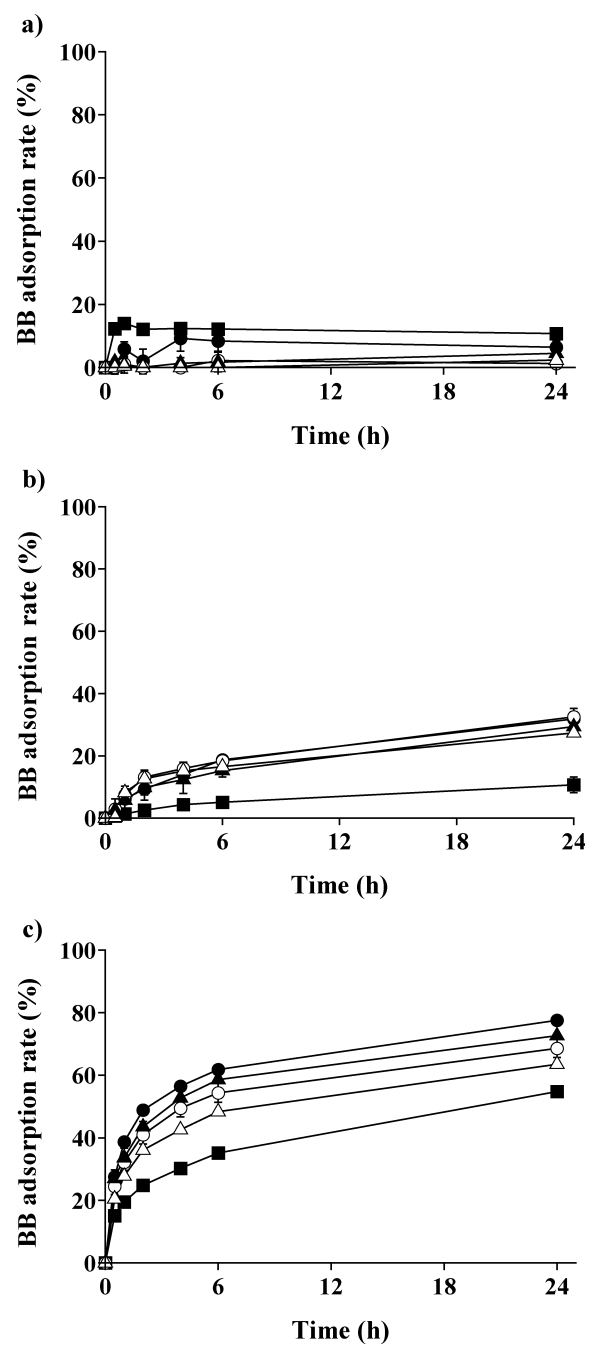

Fig. 3. Influence of SOR and MT on Brilliant Blue Adsorption Profiles of MC Films Prepared Using a) CMC, b) HPMC and c) ALG as Film Bases

MC alone, SOR (PM), O SOR (PRE), $\triangle \mathrm{MT}(\mathrm{PM}), \triangle \mathrm{MT}$ (PRE). Each point represents mean \pm S.D. $(n=3)$.
As we have proposed previously, ${ }^{13)}$ these results probably reflect non-competitive inhibition. Saccharides and polymers additives fill the pores of the MC contained in their films, taking up space which could otherwise be filled by BB. It seems likely, therefore, that the different adsorption capacities associated with the two different saccharides are probably due to saccharides' relative water solubilities. SOR, being more soluble than MT, dissolves faster, freeing MC pores and making them available to more quickly; this is why SOR did not inhibit BB adsorption as strongly as MT did. ${ }^{12)}$ Likewise, polymers' solubilities and viscosities may affect the adsorption rates of the films that contain them; this would explain why dissolved CMC and HPMC inhibited BB adsorption so much more strongly than ALG did. This could also explain the different $\mathrm{BB}$ adsorption rates exhibited by films containing PM and those containing PRE: in PRE films, more of the MC pores were filled with saccharides at the beginning of the experiment, so that it took longer for the same number of MC pores to become available.

The vertical strengths and disintegration times of $\mathrm{MC} /$ ALG films containing MC alone and those containing mixtures of MC and SOR or MT are shown in Fig. 4. The MC/
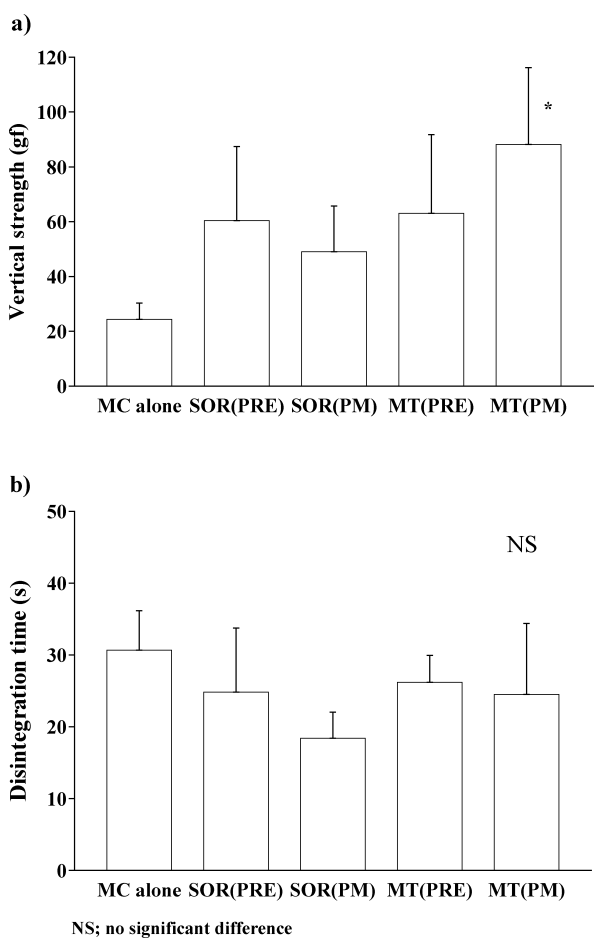

Fig. 4. Influence of SOR and MT on a) Vertical Strength and b) Disintegration Time of MC/ALG Films

Each point represents mean \pm S.D. $* p<0.05$ compared with MC alone.

Table 2. Brilliant Blue Adsorption Rates of MC Powder and MC/ALG Films Containing a Physical Mixture of MC and SOR or MT after 1, 6, and 24h of Incubation at $37^{\circ} \mathrm{C}$

BB absorption rate $(\%)^{a)}$

\begin{tabular}{cll}
\hline $1 \mathrm{~h}$ & \multicolumn{1}{c}{$6 \mathrm{~h}$} & $24 \mathrm{~h}$ \\
\hline $54.7 \pm 0.08$ & $68.0 \pm 0.61$ & $76.4 \pm 0.94$ \\
$19.5 \pm 1.05^{*}$ & $35.1 \pm 1.16^{*}$ & $54.8 \pm 1.59 *$ \\
$38.7 \pm 1.15^{*, * *, * * *}$ & $61.8 \pm 0.82^{*, * * * * * *}$ & $77.6 \pm 0.36^{* *, * * *}$ \\
$33.6 \pm 3.08^{*, * *}$ & $58.6 \pm 0.65^{* * * *}$ & $72.6 \pm 0.41^{* * * *}$
\end{tabular}

a) Mean \pm S.D. $(n=3)$. $* p<0.05$ compared with MC powder, $* * p<0.05$ compared with MC-only film, $* * * p<0.05$ compared with MC/MT-PM film. 
ALG films containing SOR or MT tended to exhibit greater vertical strengths than the $\mathrm{MC} / \mathrm{ALG}$ films without saccharides, though this difference was not statistical significant, and neither the saccharide type nor the mixture type had a clear effect on vertical strength. The disintegration times of the MC/ALG films film were not clearly affected by the addition of SOR or MT in either type of mixture.

\section{Conclusions}

The results of this study show that ALG is a useful film base for the preparation of $\mathrm{MC}$ films, and that $\mathrm{MC} / \mathrm{ALG}$ films with a high adsorption capacity, matching that of $\mathrm{MC}$ powder, can be produced by combining $\mathrm{MC}$ in a physical mixture with SOR. Furthermore, the addition of SOR or MT to MC/ALG films may increase their strength as well as improving their adsorption capacity. These findings suggested that MC-containing films could be manufactured and administered as a new dosage form, but the MC films prepared in this study contained such small amounts of MC that they could not be used clinically. Based on the results obtained in this report, we plan to investigate the potential of similar films containing more $\mathrm{MC}$ for clinical use.

\section{References}

1) Swartz C. M., Sherman A., J. Clin. Psychopharmacol., 4, 336-340 (1984).

2) Fricke R. F., Jorge J., J. Toxicol. Clin. Toxicol., 25, 421-431 (1990).

3) Makosiej F. J., Hoffman R. S., Howland M. A., Goldfrank L. R., J. Toxicol. Clin. Toxicol., 31, 381-395 (1993).

4) Coony D. O., J. Toxicol. Clin. Toxicol., 33, 213-217 (1995).

5) Tsujikawa T., Araki Y., Makino J., Uda K., Ihara T., Sasaki M., Fujiyama Y., Bamba T., J. Gastroenterol., 35, 296-298 (2000).

6) Iwata M., Takahashi T., Takahashi Y., Ito A., Machida Y., Jpn. J. Pharm. Health Care Sci., 27, 63-68 (2001).

7) Akizawa T., Koide K., Koshikawa K., Kidney and Dialysis, 45, 373388 (1998).

8) Yago K., Kuroyama S., Odori K., New Remedies \& Therapy, 47, 1417 (1997).

9) Sugihara M., Farumashia, 30, 1396-1400 (1994).

10) Yamamoto K., Onishi H., Ito A., Machida Y., Chem. Pharm. Bull., 54, 359-362 (2006).

11) Ito A., Onishi H., Yamamoto K., Machida Y., Yakugaku Zasshi, 126, 315-319 (2006).

12) Yamamoto K., Ito A., Machida Y., Chem. Pharm. Bull., 57, 10581060 (2009).

13) Yamamoto K., Onishi H., Ito A., Machida Y., Int. J. Pharm., 328, 105-111 (2007). 